MITSUBISHI ELECTRIC RESEARCH LABORATORIES

http://www.merl.com

\title{
Wireless Power Transfer with Metamaterials
}

Wang, B.; Teo, K.H.; Nishino, T.; Yerazunis, W.; Barnwell, J.; Zhang, J.

TR2011-052 April 2011

\begin{abstract}
In this paper, wireless power transfer based on resonant coupling with metamaterials is studied. We show with numerical studies that the coupling between transmitter and receiver can be enhanced, and the power transfer efficiency can be improved by metamaterials. A prototype wireless power transfer system and a metamaterial is designed and built. Experiment results prove the efficiency improvement with the fabricated metamaterial. The system with metamaterial is capable of transferring power wirelessly at roughly double the efficiency of the same system without a metamaterial.
\end{abstract}

European Conference on Antennas and Propagation (EUCAP)

This work may not be copied or reproduced in whole or in part for any commercial purpose. Permission to copy in whole or in part without payment of fee is granted for nonprofit educational and research purposes provided that all such whole or partial copies include the following: a notice that such copying is by permission of Mitsubishi Electric Research Laboratories, Inc.; an acknowledgment of the authors and individual contributions to the work; and all applicable portions of the copyright notice. Copying, reproduction, or republishing for any other purpose shall require a license with payment of fee to Mitsubishi Electric Research Laboratories, Inc. All rights reserved.

Copyright (C) Mitsubishi Electric Research Laboratories, Inc., 2011

201 Broadway, Cambridge, Massachusetts 02139 



\title{
Wireless Power Transfer with Metamaterials
}

\author{
Bingnan Wang*, Koon Hoo Teo*, Tamotsu Nishino ${ }^{\dagger}$, William Yerazunis*, John Barnwell*, Jinyun Zhang* \\ *Mitsubishi Electric Research Laboratories, \\ 201 Broadway Ste 8, Cambridge, MA 02139 USA \\ bwang@merl.com, teo@merl.com, yerazunis@merl.com, barnwell@merl.com, jzhang@merl.com \\ ${ }^{\dagger}$ Kamakura Factory, Mitsubishi Electric Corp, \\ 5-3-19-213 Koshigoe Kamakura Kanagawa 2480033 Japan \\ nishino@ieee.org
}

\begin{abstract}
In this paper, wireless power transfer based on resonant coupling with metamaterials is studied. We show with numerical studies that the coupling between transmitter and receiver can be enhanced, and the power transfer efficiency can be improved by metamaterials. A prototype wireless power transfer system and a metamaterial is designed and built. Experiment results prove the efficiency improvement with the fabricated metamaterial. The system with metamaterial is capable of transferring power wirelessly at roughly double the efficiency of the same system without a metamaterial.
\end{abstract}

\section{INTRODUCTION}

Wireless power transfer (WPT) technologies have attracted a lot of attention in recent years. The applications of WPT technologies can be very broad, from low-power consumer electronics to implanted medical devices to high-power industrial and electric vehicle applications. WPT was first pursued by Tesla over 100 years ago, however, safety, efficiency, and other issues have prevented WPT from deployment real applications in daily life. Recent research efforts have been focused on technologies based on magnetic coupling [1]-[4], as they are less sensitive to surrounding objects and safer for humans, and more suitable for the increasing demands for wireless charging of electronic devices.

With magnetic coupling or inductive coupling, very high efficiency between transmitting and receiving coils can be achieved at small distances which are less than a few centimeters [6]. That means the transmitter and receiver must be strongly coupled, with coupling coefficient usually greater than 0.9. When the transmitter and receiver are working at their resonant frequencies, the range of efficient WPT can be extended dramatically. With a much smaller coupling coefficient (usually less than 0.1), a moderate power transfer efficiency can still be obtained. High-Q resonators are required for resonant coupling to reduce losses, so that more power is coupled to receiver than lost in radiation and heat. The basic principle was discovered many years ago; however, the research and application has been quite limited. In previous studies, the required transfer distance is just a few centimeters and the required power level is relatively low [7], [8]. Recently, WPT over a distance up to 2 meters, with moderate efficiency and power level has been achieved [1]. The discovery opens up the possibility of many potential applications such as wireless charging for electric vehicles and wireless power of industrial equipments. High $\mathrm{Q}$ resonators is essential to achieve high transfer efficiency. On the other hand, the coupling coefficient is also important, as higher coupling coefficient leads to higher efficiency.

We have proposed the use of metamaterials to enhance the coupling coefficient of transmitter and receiver, and improve the WPT efficiency in [10]. In this paper, we continue the study and do experimental measurements to prove the transfer efficiency improvement. We designed and build a WPT system with a magnetic metamaterial, and we show by experimental measurements that metamaterials can be used to improve the power transfer efficiency of a WPT system based on resonant coupling.

\section{Metamaterials And WPT}

Metamaterials are usually composed of artificial structures that show peculiar electromagnetic properties such as negative refraction [9]. The elemental structures in metamaterials are typically much smaller than the wavelength and the novel electromagnetic (EM) properties of metamaterials are obtained from these elemental structures, instead of the composite materials. Macroscopic parameters, such as electric permittivity $\epsilon$ and magnetic permeability $\mu$, can then be used to describe the EM properties of metamaterials [6]. Almost arbitrary parameters can be achieved by carefully designing the elemental structures of metamaterials. Negative-index metamaterials (NIMs) are the first and one of mostly studied metamaterials. With a flat slab of NIM, negative refraction can happen at both interfaces. When an object is in front of such a slab, the propagating wave components of the object can be focused inside the NIM slab, and refocused on the other side of the slab. Moreover, evanescent wave components can be enhanced inside a NIM slab to levels similar to the levels adjacent to the original electrical conductor. Similarly, with a NIM slab, evanescent waves of an object can be restored at a distance far greater than the classical distance of $\lambda / 3$. This means, a NIM slab recovers both propagating waves and evanescent waves of an object and makes a "perfect lens" [11].

The properties of NIMs, especially evanescent wave enhancement, are of interest to us because the magnetic resonant coupling is essentially evanescent wave coupling. At resonance, EM fields are confined mostly inside the resonators, and electric energy and magnetic energy exchange periodically. 
Outside the resonators, the EM fields decay evanescently and do not carry away energy, unless coupled to the tail of the evanescent wave of another resonator. With a NIM, the amplitude of evanescent waves can be enhanced such that the distance between two resonators are virtually smaller. Thus the coupling coefficient of two resonators can be improved by a NIM slab. This is discussed and numerically studied in [10].

In general, a NIM requires both $\epsilon$ and $\mu$ to be negative, which is complicated to design and fabricate. Moreover, loss in a NIM is usually high, as it responses to both external electric field and magnetic field. However, in deep subwavelength limit, the magnetic field and electric field decouple, and only one parameter is required to be negative to achieve evanescent wave amplification [11]. This simplifies the design and fabrication process of metamaterials. For a WPT system based on coupling of magnetic fields, a magnetic metamaterial with only a negative $\mu$ can achieve the similar results to a negative $\mu$ negative $\epsilon$ NIM. Moreover, by using a singlenegative metamaterial, the loss could be reduced with simplified structures.

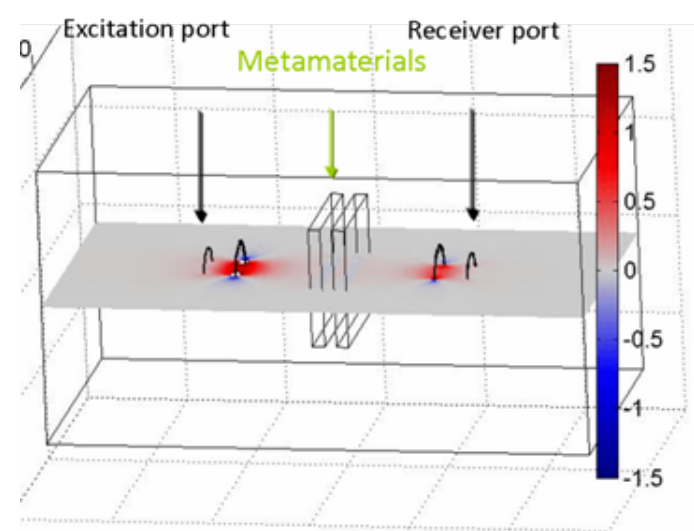

Fig. 1. Simulation result of magnetic field distribution of a resonant WPT system with metamaterials.

Fig. 1 shows the evanescent wave enhancement by a magnetic metamaterial, with $\mu=-1$ in a WPT system by resonant coupling of two coil resonators. Magnetic field distribution is plotted in the figure. At the two coils, strong field is localized; field decays away from the coils. In the middle of the system, magnetic metamaterials enhance the evanescent wave, and improve the coupling between transmitter and receiver.

Due to the increased coupling between transmitter and receiver, the power transfer efficiency can be improved. Fig. 2 shows the simulated power transfer efficiency of a WPT system with and without metamaterials, as a function of excitation frequency. From the figure, we can see that the power transfer efficiency is much higher at resonant frequency. With metamaterials, the simulation shows that the peak efficiency is increased from $35 \%$ to about $50 \%$. The simulations are done in COMSOL, a finite-element based software. In the simulations, an excitation port is set at the loop antenna (shown in Fig. 1), and another port is used at the receiving loop antenna for power pickup. Efficiency is measured by

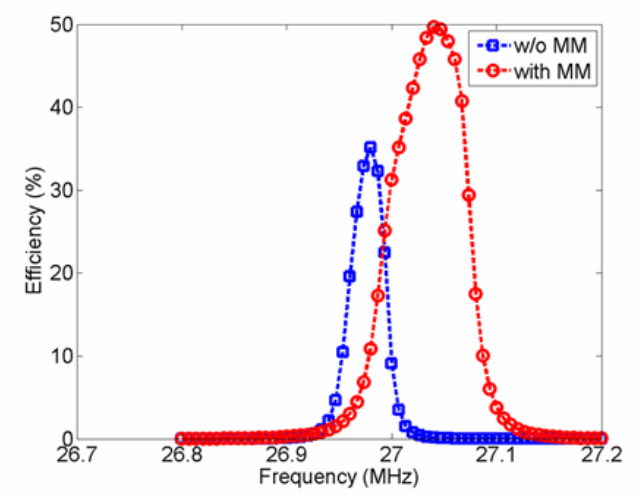

Fig. 2. Simulated power transfer efficiency of a WPT system with and without metamaterials. The system configuration in simulation is shown in Fig. 1

calculating the power ratio at receiving and transmitting ports. The excitation frequency is changed and efficiency is recorded at each frequency and plotted in Fig. 2. The figure also shows a slight peak efficiency shift, which is due to the coupling of the metamaterial and the rest of the WPT system. This can be easily fixed by frequency tuning for the system.

\section{SYSTEM DESIGN AND FABRICATION}
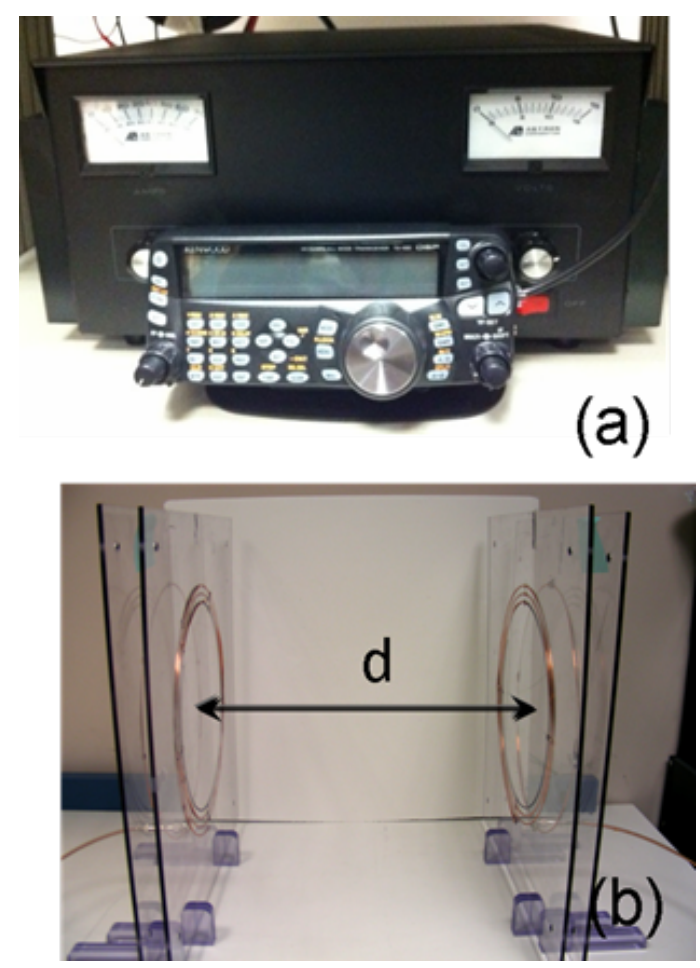

Fig. 3. (a) The HF transceiver and power amplifier. (b) The fabricated WPT system without metamaterial.

In this section, we present our work on the design and fabrication of a WPT system with metamaterial. Our experimental apparatus operates at the ISM band centered at $27.12 \mathrm{MHz}$. A modified Kenwood TS-480 serves as master oscillator and 
power amplifier, capable of providing up to $200 \mathrm{~W}$ of RF sine wave power as our HF power supply [Fig. 3(a)]. The power supply is connected to a loop antenna, which is inductively coupled to a first resonant coil. The resonant coil is coupled to a second coil, which is inductively coupled to a receiving loop antenna [Fig. 3(b)]. Power is extracted from the receiving loop antenna by a resistive load (light bulb). A metamaterial is used to place in between the transmitter and receiver for efficiency improvement. The loop antennas are copper wire loops with $20 \mathrm{~cm}$ radius. The two coils are planar spirals of 12-Gauge copper wires. The spirals have 3 turns with outer radius $20 \mathrm{~cm}$ and spacing between neighboring turns $1.5 \mathrm{~cm}$.

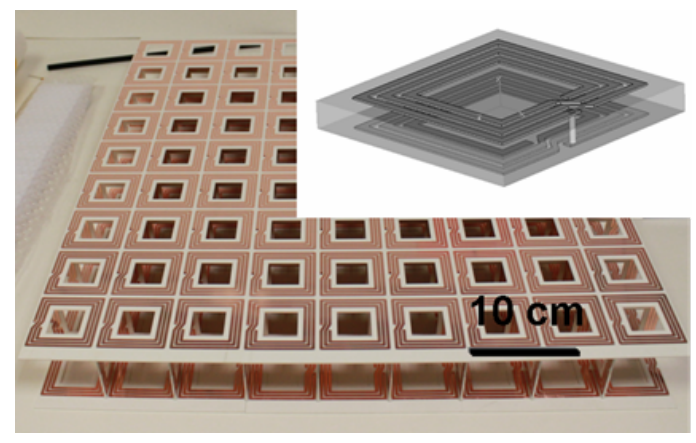

Fig. 4. The fabricated metamaterial. The structure of the unit cell is shown in the inset.

Different than near-field imaging, the metamaterial in WPT needs to have very low loss, due to the power transfer efficiency requirement. We use Rogers RO4003C board as substrate material due to its low dissipation factor. Copper patterns are used for metallic structures in the metamaterial. The design of the metamaterial structure is chosen to meet the low loss, compact size and high power handling requirements. As shown in Fig. 4, the metamaterial is a 3D arrangement of double-side square spiral structures. The double-side square spiral serves as a building block of the metamaterial, and gives a strong resonant response to external magnetic field around $24 \mathrm{MHz}$. The strong response comes from the resonance of the structure, which can be effectively considered as an LC resonator, where the inductance comes from the metal wire structure, and the capacitance comes mainly from the "plate capacitor" due to the two sides of metal structure. At our working frequency of $27.12 \mathrm{MHz}$, this metamaterial has an effective $\mu$ very close to -1.0 without needing per-resonator manual adjustments, and with easy manufacturability, low loss, and compact size. In terms of wavelength to unit cell ratio, the current design is about 170 , while conventional split-ring resonator is around 10. The magnetic metamaterial is fabricated with standard PCB processing technology. The structures are assembled in a planar slab shape, with 9 by 9 elements in plane. The size of the fabricated metamaterial is $58.8 \mathrm{~cm}$ by $58.5 \mathrm{~cm}$ by $6.5 \mathrm{~cm}$.

\section{EXPERIMENT RESULTS}

The fabricated system are used to transfer power wirelessly to a $40 \mathrm{~W}$ light bulb. In the following measurements, the

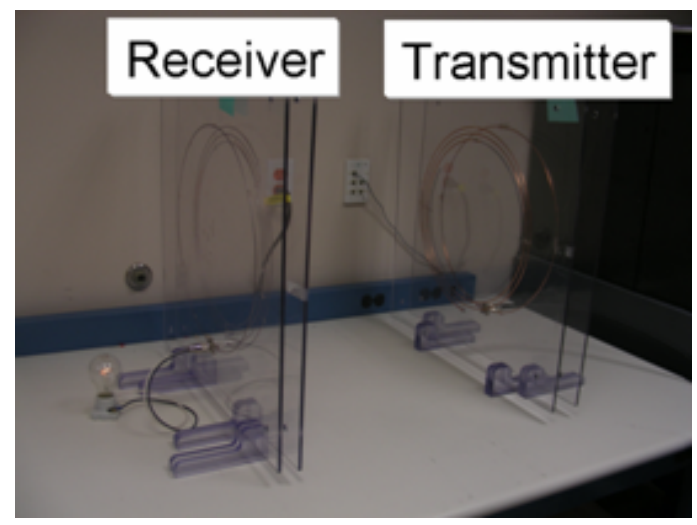

Fig. 5. Power transfer to a light bulb without metamaterial.

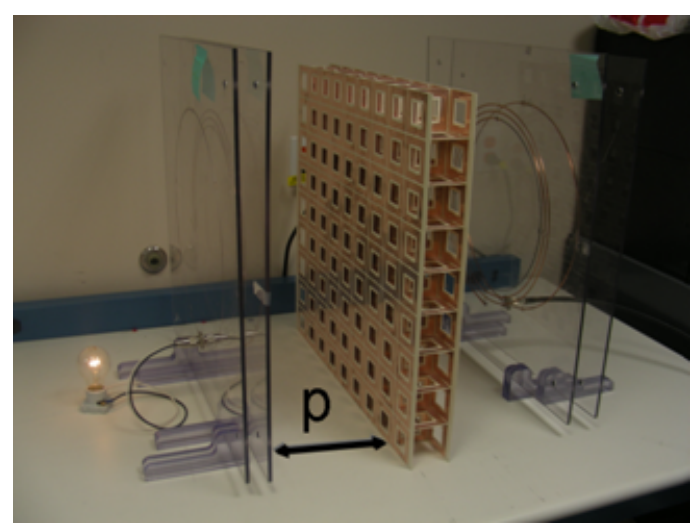

Fig. 6. Power transfer efficiency to a light bulb with metamaterial.

distance between transmitting coil and receiving coil is set to be $d=50 \mathrm{~cm}$. The $\mathrm{HF}$ transceiver is set to have a transmitting frequency of $27.12 \mathrm{MHz}$ and provide $80 \mathrm{~W}$ power to the transmitting loop antenna, which is coupled to the first coil. The light bulb is connected to the receiving loop antenna, which is coupled to the second coil. The power transfer efficiency can be observed from the brightness of the light bulb (and by RF voltmeter, not shown). The WPT system without metamaterial is shown in Fig. 5; the light bulb glows but not bright. The WPT system with metamaterial slab is shown in Fig. 6; the light bulg is much brighter compared with the system without metamaterial. This shows the power transfer efficiency is improved with the metamaterial. Further more, the fabricated metamaterial is capable of handling such high power level.

The efficiency was also measured more accurately but at a much lower power with an Agilent N5230A PNA network analyzer. The two ports of the network analyzer are connected to the two loop antennas. S-parameters are measured and the power transfer efficiency is estimated by $\left|S_{21}\right|^{2}$. The distance $d$ is still set to be $50 \mathrm{~cm}$. The efficiency as a function of frequency is plotted in Fig. 7 for different system configurations. The curve with label "original" is efficiency of system without metamaterial; The rest are measurements for the system with the metamaterial slab at different positions. The position $p$ is 


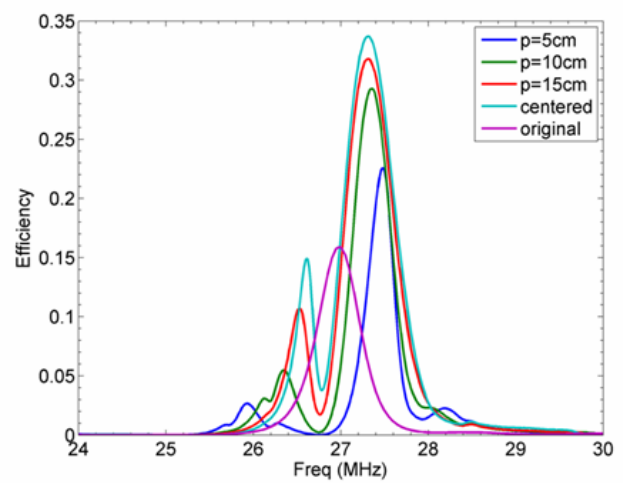

Fig. 7. Simulation result of magnetic field distribution of a resonant WPT system with metamaterials.

the distance between transmitting coil and the metamaterial slab. The curve with label "centered" is efficiency of system with the metamaterial slab at the center of transmitting and receiving coils. The peak efficiency of the original system is about $16 \%$; when the metamaterial slab is used, the system efficiency is increased by an amount depending on the metamaterial slab position. When the metamaterial slab is at the center, the efficiency has a peak value of $34 \%$, which is almost twice as high as the original system. At other locations, the efficiency improvement is not as high. This is primarily because the field strength is lowest at the center, and smaller field strength in metamaterial makes power loss due to the metamaterial lower. At other locations, the power loss due to the metamaterial is higher, thus less power delivered.

\section{CONCLUSIONS}

In conclusion, we studied wireless power transfer based on resonant coupling of evanescent waves. We proposed and investigated the use of metamaterials in wireless power transfer. Numerical simulations showed that the coupling can be enhanced between resonators with metamaterials, and power transfer efficiency can be improved in a wireless power transfer system. We also designed and built an experimental system with a magnetic metamaterial. Experimental results show that the power transfer efficiency can be improved significantly with metmaterials. This work also shows that, other than communication and other low-power applications, metamaterials can also be used in high-power energy applications.

\section{ACKNOWLEDGEMENTS}

The authors are thankful to Prof. David R. Smith, Da Huang, and Dr. Yaroslav Urzhumov, all at Duke University, for stimulating discussions on metamaterial and wireless power transfer.

\section{REFERENCES}

[1] A. Kurs, A. Karalis, R. Moffatt, J. D. Joannopoulos, P. Fisher and M. Soljiacic, "Wireless power transfer via strongly coupled magnetic resonances," Science, 317, 83 (2007)
[2] Z. N. Low, R. A. Chinga, R. Tseng and J. Lin, "Design and Test of a High-Power High-Efficiency Loosely Coupled Planar Wireless Power Transfer System," IEEE Transactions on Industrial Electronics, 56, 1801 (2009)

[3] B. L. Cannon, J. F. Hoburg, D. D. Stancil and S. C. Goldstein, "Magnetic Resonant Coupling As a Potential Means for Wireless Power Transfer to Multiple Small Receivers," IEEE TRANSACTIONS ON POWER ELECTRONICS, 24, 1819, (2009)

[4] G. A. J. Elliott, S. Raabe, G. A. Covic, and J. T. Boys, "Multiphase Pickups for Large Lateral Tolerance Contactless Power-Transfer Systems," IEEE TRANSACTIONS ON INDUSTRIAL ELECTRONICS, 57, 1590 (2010)

[5] N. Shimokura, N. Kaya, M. Shinohara, and H. Matsumoto, "point-topoint microwave power transmission experiment" Electrical Engineering in Japan 120 (1), 33-39 (1997)

[6] J. C. Schuder, H. E. Stephenson and J. F. Townsend, "High-level electromagnetic energy transfer through a closed chest wall," Inst. Radio Engrs. Int Conv. Record, 9, 119 (1961)

[7] N. de N. Donaldson and T. A. Perlins, "Analysis of resonant coupled coils in the design of radio frequency transcutaneous links," Med. \& Biol. Eng. \& Comput., 21, 612 (1983)

[8] R. Puers, K. V. Schuylenbergh, M. Catrysse and B. Hermans, "Wireless inductive transfer of power and data," Analog Circuit Design, Springer p395 (2006)

[9] D. R. Smith, J. B. Pendry, and M. C. K. Wiltshire, "Metamaterials and negative refractive index," Science, 305, 788 (2004)

[10] B. Wang, T. Nishino and K. H. Teo, "Wireless power transmission efficiency enhancement with metamaterials," in Proceedings of the IEEE International Conference on Wireless Information Technology and Systems (ICWITS'10), Honululu, Hawai'i, 28. August - 03. September, 2010

[11] J. B. Pendry, "Negative refraction makes a perfect lens," Phys. Rev. Lett. 85, $3966(2000)$ 